\title{
Fracturas diafisiarias de fémur en pacientes pediátricos del Centenario Hospital Miguel Hidalgo. Estudio epidemiológico de cinco años
}

\author{
Reyes-Hernández Lydia Alessandra*, Barajas-Salcedo Gerardo**, Cervantes-Gudiño Jorge Enrique***
}

\section{Resumen}

- Las fracturas diafisiarias de fémur comprenden el $4 \%$ de los

- huesos largos en pacientes pediátricos. Es la lesión que más

- necesita hospitalización. Las principales causas son caídas

- y accidentes de tránsito; sin embargo, en niños menores de

- edad, el abuso debe ser considerado. El tratamiento de estas

- fracturas está determinado por una serie de factores que in-

- cluyen edad y peso, características de la fractura, mecanismo

- de lesión, situación social de la familia y comorbilidades del

- paciente. El estudio es retrospectivo y descriptivo de cinco

- años con el objetivo de conocer la prevalencia, el sitio ana-

- tómico más afectado, mecanismo de lesión y tratamiento

- otorgado. Se recolectaron 52 pacientes, de los cuales predo-

- minó 3:1 el sexo masculino, la edad promedio fue de 6.88

- años. El grupo de edad que mayor porcentaje representó fue

- los menores de 7 años, tipo de trazo de fractura que más

- se presentó fue el transverso. El 88.5 \% fueron fracturas

- no expuestas de las cuales el $60.86 \%$ recibió manejo con-

- servador, mientras que las fracturas expuestas el 100\% fue

- tratada mediante tratamiento quirúrgico. El 70 \% de los pa-

- cientes no presentó complicación alguna y todos egresaron

- por mejoría. La prevalencia que encontramos fue de 3 por

- cada 1000 pacientes que llegaron al servicio de urgencias

- pediátricas por lesiones traumáticas y se concluye que en

- el servicio de ortopedia pediátrica cada paciente recibió un

- tratamiento individualizado, y se esperar un pronóstico bue-

- no para la extremidad afectada y para la vida. LUXMÉDICA,

- AÑO 14, NÚMERO 40, ENERO-ABRIL 2019, PP 17-27.

Palabras clave: fracturas de fémur, prevalencia, complica-

\section{Abstract}

Diaphyseal femur fractures are the orthopedic injury in children that most frequently requires hospitalization. In general, its causes are falls and traffic accidents. The treatment is determined by a series of factors, including the age and weight of the patient, the characteristics of the fracture, the mechanism of injury, as well as the social situation of the family and the patient's comorbidities. Objective: to know the prevalence of diaphyseal femur fractures in pediatric patients of Centenario Hospital Miguel Hidalgo. Methods: retrospective, descriptive and transversal study. Fifty-two patients were included, male sex was predominant, the average age was 6.8 \pm 5.4 years. $88.5 \%$ were closed fractures, of which $60.86 \%$ received conservative treatment with glass fiber spica cast; for the open fractures, $100 \%$ received surgical treatment: $50 \%$ were treated by closed reduction and intramedullary nailing with unreamed femoral nail (UFN), 33.4\% with closed reduction plus external fixator and only $16.6 \%$ with the closed reduction technique plus intramedullary nailing with titanium elastic nail system (TENS). Seventy percent of the patients did not present any complication, and all were discharged referring improvement. The prevalence we found was 3 per 1,000 patients who arrived at the pediatric emergency room for traumatic injuries and we concluded that in the pediatric orthopedic department each patient received an individualized treatment, expecting a good prognosis for the affected extremity and for life. LUXMÉDICA, AÑO 14, NÚMERO 40, ENERO-ABRIL 2019, PP 17-27.

Keywords: femur fractures, prevalence, complications 


\section{Introducción}

Las fracturas de la diáfisis femoral comprenden cerca del $4 \%$ de todos los huesos largos en pacientes pediátricos. ${ }^{1}$ Se calcula una incidencia de 15-25 casos por cada 100,000 pacientes menores de los 16 años; representa la segunda localización más frecuente de la extremidad inferior ${ }^{2,3}$ y es la lesión ortopédica más común entre los niños que requieren hospitalización. ${ }^{4}$ Las causas más comunes de fracturas de la diáfisis femoral son caídas y accidentes de tránsito; sin embargo, en niños menores de edad, el abuso debe ser considerado. Un método común utilizado para clasificar las fracturas diafisiarias de fémur en niños es el descriptivo, de acuerdo con el trazo de la fractura: 1) transversal, espiral u oblicuo; 2) en cuña y multifragmentada; y 3) fracturas expuestas o fracturas cerradas (en las cuales el hueso no tuvo contacto con el medio externo. ${ }^{5}$ Las fracturas expuestas se subclasifican adicionalmente, de acuerdo con Gustilo y Anderson en Tipo I a III en función al tipo de energía, la contaminación y la alteración de los tejidos blandos. ${ }^{6}$ El tipo III de la clasificación de Gustilo y Anderson se subdivide a su vez como A, $B$ y $C$, en función de la severidad de la lesión de los tejidos blandos, la energía del trauma, la eliminación del periostio y la necesidad de reconstrucción vascular. ${ }^{7}$ Según la clasificación pediátrica del $A r-$ beitsgemeineschaft fur Osteosynthesefragen (AO) de las fracturas de huesos largos, ${ }^{8}$ las fracturas del eje femoral se clasifican en la categoría 32-D. Las subcategorías 32-D4. ${ }^{1}$ (transversal completa con una oblicuidad de $30^{\circ}$ o menos) y 32-D 5.1 (oblicuo largo o espiroideo de más de $30^{\circ}$ ) son fracturas simples. Las fracturas de cuña y de múltiples fragmentos se subcategorizan en 32-D 4.2 (multifragmentaria transversal $30^{\circ}$ o menos) y 32 D 5.2 (multifragmentaria oblicua o espiroidea más de $30^{\circ}$ ). La inestabilidad de la fractura puede ser el resultado de la geometría de la fractura oblicua / espiral, la conminución y la lesión de los tejidos blandos. El tratamiento de las fracturas femorales en niños y adolescentes está determinado por una serie de factores, que incluyen la edad y el peso del paciente, las características de la fractura, el mecanismo de lesión, así como la situación social de la familia y las comorbilidades del paciente..$^{9}$ Las opciones de tratamiento se relacionan directamente con la edad del paciente y el tipo de fractura. ${ }^{10}$ En el caso de los pacientes menores de cinco años, existe un debate continuo entre los cirujanos que prefieren los métodos conservadores ${ }^{11}$ y los que apoyan un enfoque quirúrgico. ${ }^{12}$ Como consecuencia, las pautas de la Academia Estadounidense de Cirugía Ortopédica (AAOS, por sus siglas en inglés) recomiendan la colocación temprana de espica o la tracción con yeso retardado para niños de seis meses a cinco años. ${ }^{13} \mathrm{~A}$ diferencia de las directrices estadounidenses, la Sociedad Alemana de Cirugía Pediátrica, recomienda que las fracturas de la diáfisis femoral se traten con clavos intramedulares elásticos estables en niños mayores de tres años. ${ }^{14}$ Esto se basa en una movilización más temprana para evitar los efectos negativos de la inmovilización. 
Las fracturas desplazadas se pueden reducir utilizando técnicas cerradas o abiertas. Los principales tipos de tratamiento conservadoras son:4

- Arnés Pavlik: es un cabestrillo con correas para el pecho, los hombros y las piernas, comúnmente usadas para la displasia del desarrollo de la cadera. Es útil para inmovilizar la fractura en flexión y abducción, y es cómoda para niños menores de seis meses de edad. El tiempo necesario de inmovilización suele ser de cuatro semanas, y la deformidad en antecurvatum y acortamiento que habitualmente aparece suele ir desapareciendo en controles sucesivos gracias a la gran capacidad de remodelación existente a estas edades.

- La tracción de Bryant: con la cadera flexionada a $90^{\circ}$ y la rodilla en extensión, el peso debe permitir despegar las nalgas de la cama. Se realiza un control radiológico a las 48 horas para verificar la reducción y el acortamiento. Una vez se haya conseguido un callo suficiente (alrededor de tres semanas), se coloca en la consulta un yeso pelvi-pédico, con una duración de seis semanas. La flexión de la cadera es alrededor de $60^{\circ}$, en ligera abducción, con la rodilla en $45^{\circ}$ de flexión. ${ }^{15}$ Se recomienda utilizar $0,5 \mathrm{Kg}$ por cada año de edad y se requiere una semana de tracción por cada año de edad como norma general. ${ }^{16}$

- Fibra de vidrio Espica: se aplica un aparato circular de fibra de vidrio desde la región subcostal hasta los dedos del pie del lado afectado, con o sin tracción preliminar. Una posición segura y efectiva es $30^{\circ}$ de abducción, $30^{\circ}$ a $40^{\circ}$ de flexión y rotación externa en la cadera. Se realiza un seguimiento semanal o quincenal, dependiendo de la estabilidad de la fractura, mediante radiografías anteroposterior y lateral hasta la formación de callo óseo como para permitir la retirada del yeso desde las seis a doce semanas aproximadamente. En cuanto al tratamiento quirúrgico se recomienda: ${ }^{17}$

a. Placas atornilladas: se aplica desde hace décadas con unos excelentes resultados inmediatos. La necesidad de una reducción a cielo abierto y una segunda intervención para llevar a cabo el retiro del material conlleva una nueva desperiostización, lo cual puede provocar un excesivo fenómeno de hipercrecimiento femoral, a veces de hasta 4 $\mathrm{cm}$. Una buena indicación de este método de osteosíntesis son las fracturas subtrocantéricas, de difícil manejo con métodos ortopédicos, así como con otros métodos quirúrgicos.

b. Clavo intramedular rígido: empezó a aplicarse en la población pediátrica a raíz de los buenos resultados obtenidos en adultos con este tipo de clavos. Al igual que con el método anterior, los resultados inmediatos son excelentes y además no precisa de la apertura del foco de fractura; pero, estudios a largo plazo han podido comprobar que la entrada del clavo a través de la punta del trocánter mayor o de la fosita piriforme provoca significativas alteraciones del crecimiento del fémur proximal (coxa valga, detención del crecimiento del trocánter mayor y adelgazamiento del diámetro del cuello femoral). ${ }^{18}$ Además, y lo que es más importante, el análisis de casos recogidos en la bibliografía ha mostrado que el riesgo de necrosis de la cabeza femoral por lesión de la vascularización al introducir el clavo a través de la fosita piriforme es del 1-2\%. ${ }^{19} \mathrm{La}$ mayoría de los autores lo recomiendan en pacientes que estén próximos o ya hayan terminado el crecimiento, a partir de los 13 años. En la actualidad, nuevos modelos de clavos con una angulación en su porción proximal similar a los clavos tibiales o humerales se están empleando en niños mayores de diez años 
con el fin de evitar los vasos retinaculares del cuello femoral entrando en la cavidad medular femoral tras abordaje trocantérico o subtrocantérico.

c. Enclavado intramedular elástico (TENS): es el método de elección para el tratamiento de las fracturas diafisiarias desplazadas. Se utilizan clavos de titanio o acero de diferentes calibres en función del diámetro de la cavidad medular diafisiaria. Como fórmula de fácil retención se puede medir el diámetro de la cavidad medular y multiplicarla por el coeficiente 0,4 con el objetivo de ocupar el $80 \%$ del espacio medular en el 1/3 medio femoral. El mayor diámetro del clavo elástico confiere mayor resistencia a la deformación del foco de fractura. Los clavos son introducidos a distancia del foco de fractura, respetando las fisis. Los clavos tienen una configuración curvada previamente establecida de manera que siempre tienen, al menos, tres puntos de apoyo en el hueso confiriendo al montaje una estabilidad elástica. Este procedimiento obtiene muy buenos resultados en fracturas transversales u oblicuas cortas que asientan en el tercio medio de la diáfisis. No obstante, en las fracturas conminutas o con gran inestabilidad en el foco fracturario, la síntesis intramedular elástica puede que no sea suficiente para controlar el acortamiento, la angulaciones axiales o rotacionales y puede precisar la colocación adicional de un yeso pelvipédico durante un período de tres a cinco semanas.

d. Fijación externa: cuenta con unas indicaciones clásicas en fracturas diafisarias expuestas para control de daños, paciente con politrauma, fracturas conminutas, fracturas con pérdida ósea im- portante. En las fracturas metafiso-diafisiarias distales la colocación de fijador puenteando la fisis temporalmente permite estabilizar y controlar la fractura hasta su curación. En cuanto al tipo de clavos que se deben emplear, deben ser de 5-6mm, excepto en niños pequeños que tengan un diámetro diafisiario femoral inferior a $2 \mathrm{~cm}$. En esta situación, el calibre del clavo recomendado debe ser de 4-5mm.

Dentro de las complicaciones tempranas, difieren bastante en su frecuencia con respecto al adulto. El "tromboembolismo" es excepcional, pero se han descrito en el período puberal. En estos casos deberemos establecer pautas profilácticas con antiagregantes plaquetarios o heparinas de bajo peso molecular. ${ }^{17}$ La infección $(<2 \%)$ se observa ocasionalmente cuando se trata de una fractura expuesta o hay un gran daño a los tejidos blandos o cuando se requirió abrir el foco fracturario para reducir la fractura. La osteomielitis a estas edades puede contribuir desfavorablemente en el hipercrecimiento de la extremidad. Las complicaciones tardías se presentan a lo largo del proceso de consolidación o en la evolución posterior. Éstas se encontrarán influenciadas por las características de la fractura, de la persona que presenta dicha lesión y por el sistema de tratamiento elegido. Las complicaciones generales del tratamiento quirúrgico son: mala unión, acortamiento, infección en el sitio de inserción de los pines, arresto fisiario (usualmente por mala colocación de los clavos de la tracción), úlcera por decúbito, síndrome compartimental, úlceras por presión, lesión nerviosa, refractura y lesiones de la piel con la sierra al retirar yeso. ${ }^{21}$

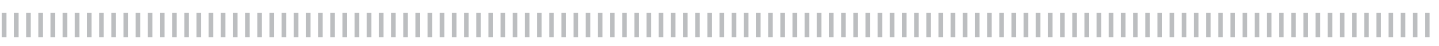

\section{Material y Métodos}

Se realizó un estudio de tipo observacional y descriptivo con diseño retrospectivo y direccionalidad longitudinal en pacientes pediátricos del Centenario Hospital Miguel Hidalgo en un periodo de cinco años que 
abarca del 2013 al 2017. El objetivo fue conocer la prevalencia de las fracturas diafisiarias de fémur en pacientes pediátricos del Hospital Centenario Miguel Hidalgo, el sitio anatómico más afectado, mecanismo de lesión, tipo de tratamiento otorgado dependiendo la edad del paciente: conservador o quirúrgico y las complicaciones suscitadas. El tipo de muestreo utilizado fue no probabilístico, por conveniencia. Los criterios de inclusión fueron la edad pediátrica y el diagnóstico de fractura de fémur, y se analizaron los datos clínicos y radiográficos de los pacientes seleccionados. El total de pacientes ingresado al estudio fue de 52. Se realizó una base de datos en el programa SPSS versión 21.0 para realizar la estadística descriptiva utilizando medidas de tendencia central, medidas de dispersión, tablas de frecuencias y porcentajes, además de análisis univariados y bivariados.

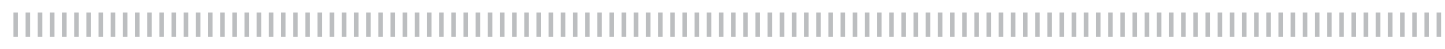

\section{Resultados}

De 17,500 pacientes atendidos en el servicio de urgencias pediátricas del Centenario Hospital Miguel Hidalgo por lesiones traumáticas durante el período de estudio, 52 corresponden a fractura de fémur, obteniéndose una prevalencia de 3 fracturas de fémur por cada 1000 pacientes que acuden por lesiones traumáticas. En cuanto a la distribución por sexo, se observó mayor frecuencia en el sexo masculino con $36 \mathrm{ca}$ sos, representando el $69.2 \%$, en el sexo femenino fueron 16 casos, representando del 30.8\%, dando una relación de 3:1 respecto al sexo masculino vs sexo femenino. En cuanto a la edad, se observó una gran dispersión al encontrar cinco pacientes menores de un año hasta una edad máxima de 18 años, con una media de 6.88 años y una desviación estándar de \pm 5.48 . Al representarlos por grupo de edad, podemos observar que el 54\% lo ocupan menores de 7 años, y los pacientes de 7 años a 13 años representan un $21 \%$, mientras que los mayores de 13 años representan el $25 \%$ (figura 1 ).

\section{Representación por grupos de edad $\mathbf{n}=52$}

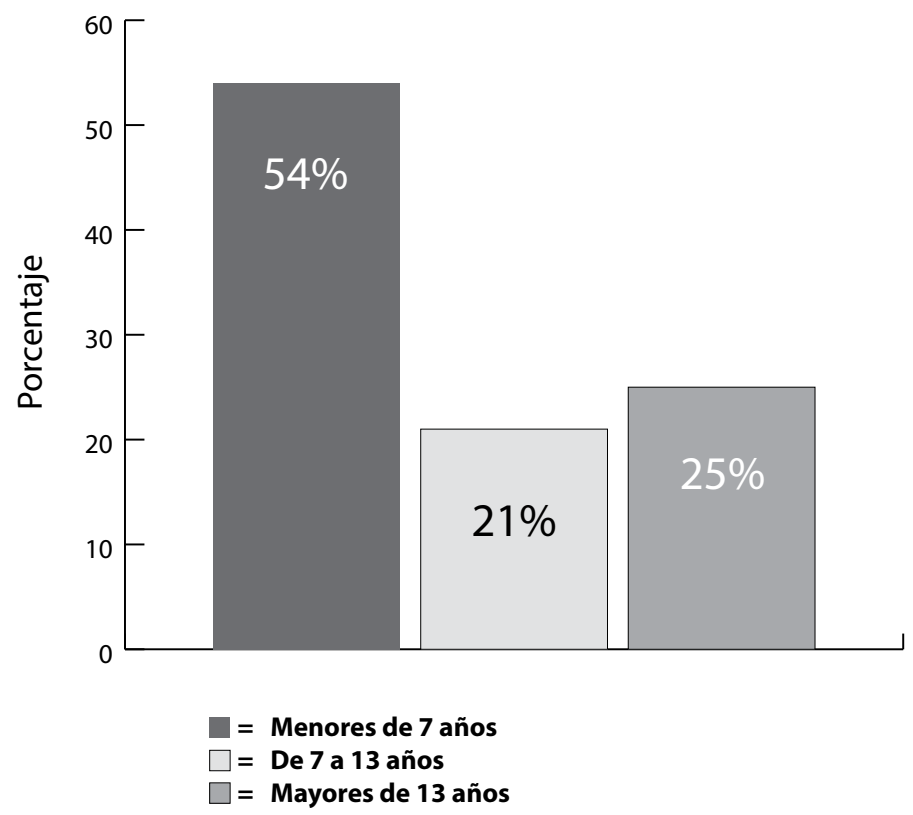

Figura 1. Distribución por edad de los pacientes pediátricos con diagnóstico de fractura de fémur del CHMH 2013-2017 
Los tres mecanismos de lesión que más se registraron fueron caída de la propia altura en un $23 \%$, seguida de caída de motocicleta en un $15 \%$ y por atropellamiento en un $12 \%$, mientras que el otro $50 \%$ lo representan diferentes tipos de mecanismo (figura 2).

\section{Representación por mecanismo de lesión $\mathbf{n = 5 2}$}

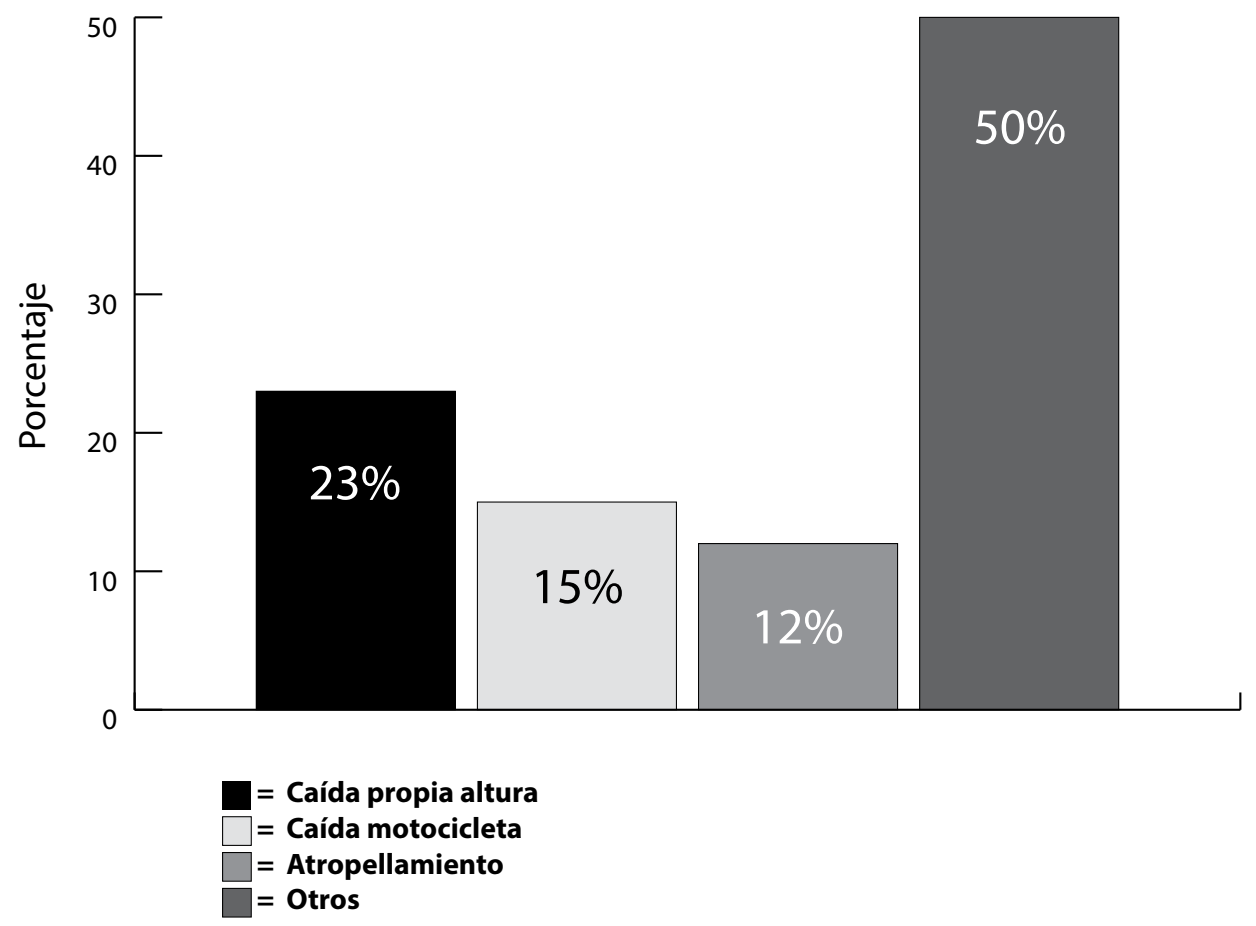

Figura 2. Distribución de los mecanismos de lesión de las fracturas de fémur en pacientes pediátricos del CHMH 2013-2017

La extremidad más afectada en nuestro grupo de estudio fue la izquierda con más del $50 \%$, mientras que la derecha se reporta en un $46 \%$ y solamente el $3 \%$ presentó fractura bilateral. Sólo el 11.5 $\%$ de los pacientes presentó fracturas expuestas y la mayor parte representada por el $88.5 \%$ fue no expuestas. Dentro de las expuestas, $66.7 \%$ se clasificó como grado
I, mientras que el $33.3 \%$ fue clasificado como grado III A.

Según las características del trazo de la fractura, observado en las placas radiográficas, se encontró que el de mayor frecuencia fue el transverso en el $50 \%$ de los casos, seguido del oblicuo en un $23 \%$, el espiroideo en un $19 \%$, el de cuña en el $6 \%$ y el multifragmentado en el $2 \%$ de los pacientes. 


\section{Tabla I}

Tipo de tratamiento por grupo de edad que recibieron los pacientes que ingresaron al estudio

\begin{tabular}{|lll}
\hline Grupo de edad & Tratamiento & \\
\hline Menores de 7 años $(\mathrm{n}=28)$ & Conservador (89\%) & Férula muslo-podálica (2 pacientes) \\
\hline & & Fibra de vidrio tipo espica (23 pacientes) \\
\hline Quirúrgico (10.71\%) & $\begin{array}{l}\text { Reducción cerrada más enclavado centro-medular } \\
\text { con clavos TENS (3 pacientes) }\end{array}$ \\
\hline 7-13 años ( $\mathrm{n}=11)$ & Férula muslo-podálica ( 1 paciente) \\
\hline & Conservador (27,27\%) & Fibra de vidrio tipo espica (2 pacientes) \\
\hline Quirúrgico 72.72\% & $\begin{array}{l}\text { Reducción cerrada más enclavado centromedular } \\
\text { con clavos TENS (5 pacientes) }\end{array}$ \\
\hline Mayores de 13 años ( $\mathrm{n}=13)$ & $\begin{array}{l}\text { Reducción abierta más fijación interna con placa (3 } \\
\text { pacientes) }\end{array}$ \\
\hline & $\begin{array}{l}\text { Reducción cerrada más enclavado centromedular } \\
\text { con clavos TENS (3 pacientes) }\end{array}$ \\
\hline & $\begin{array}{l}\text { Reducción cerrada más enclavado centromedular } \\
\text { con clavo UFN (7 pacientes) }\end{array}$ \\
\hline & $\begin{array}{l}\text { Reducción cerrada más colocación de fijador exter } \\
\text { no (2 pacientes) }\end{array}$ \\
\hline & $\begin{array}{l}\text { Reducción cerrada más fijación percutánea con cla } \\
\text { villos Steinman (1 paciente) }\end{array}$ \\
\hline
\end{tabular}

El tercio medio del fémur fue el afectado mayormente con un $65.4 \%$, seguidos del tercio distal en un $19.2 \%$ y tercio proximal en un $15.4 \%$. En cuanto al tratamiento otorgado, el $54 \%$ fue de tipo conservador, las cuales se manejaron de forma ambulatoria con vigilancia en consulta externa mediante radiografías seriadas y el $46 \%$ fue sometido a manejo quirúrgico, siendo necesaria su hospitalización para su manejo integral y vigilancia estrecha de las complicaciones que pudieran suscitarse (tabla 1).

A continuación se muestran algunas imágenes que muestran el tipo de fractura, el tratamiento otorgado y la evolución de algunos de los pacientes que ingresaron al protocolo.

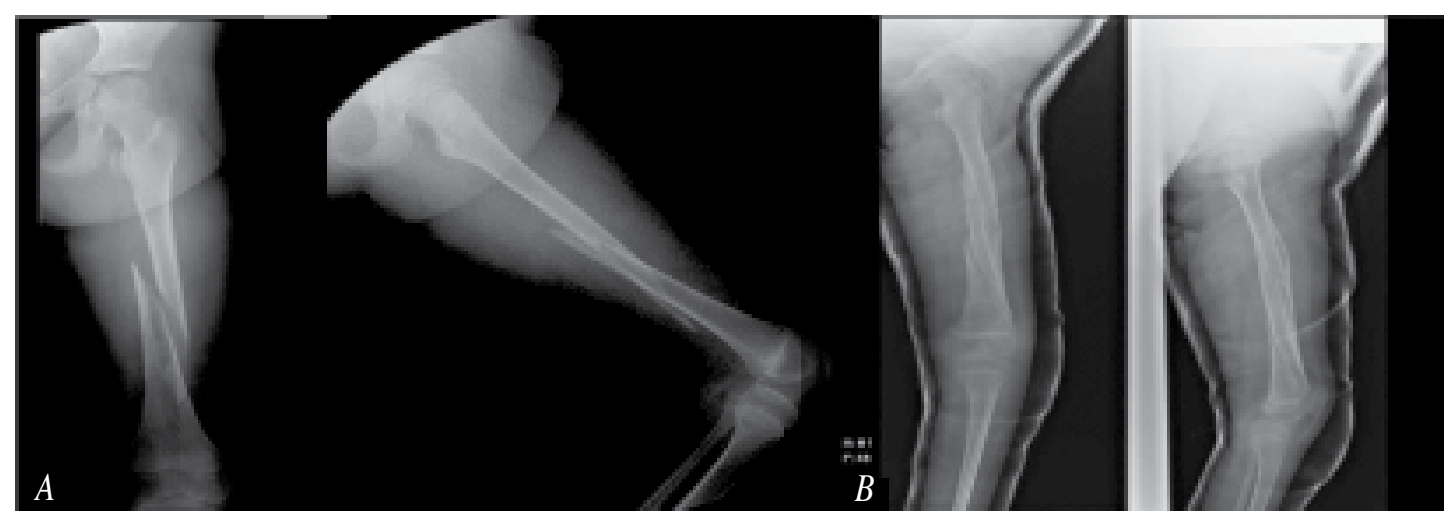

Figura 3. Panel A: radiografía antero-posterior y lateral de fémur izquierdo. Paciente femenino de cuatro años de edad, con fractura diafisaria media, trazo simple espiroideo. Panel B: radiografía antero-posterior y lateral de fémur izquierdo inmovilización con fibra de vidrio espica de cinco semanas de evolución. 

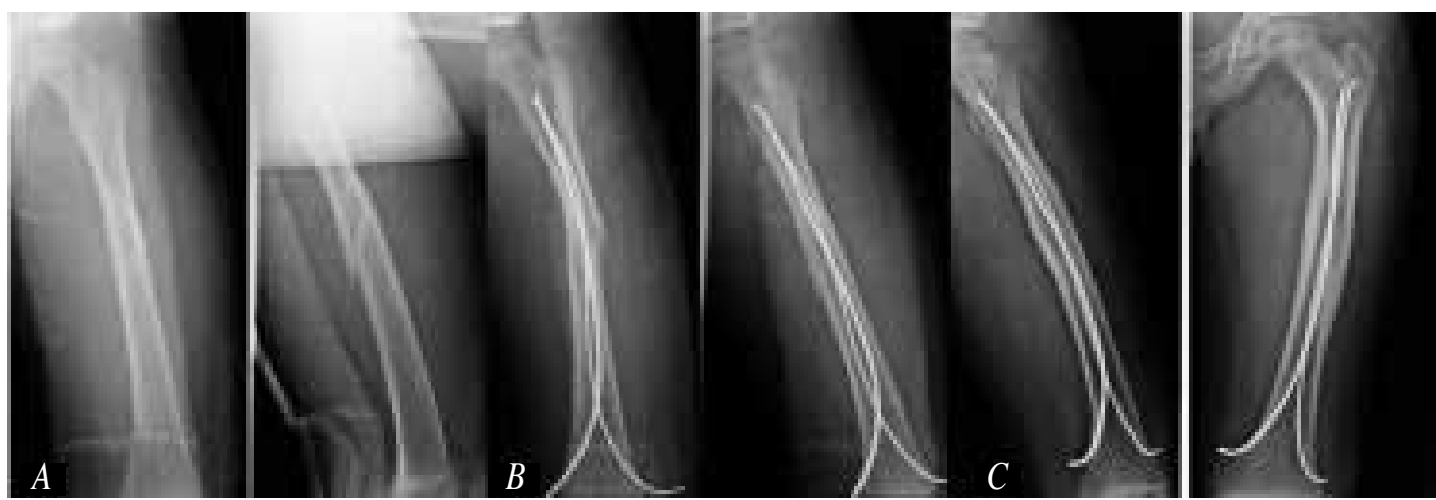

Figura 4. Panel A: radiografía antero-posterior y lateral de fémur izquierdo. Paciente masculino de 10 años de edad, con fractura diafisaria proximal, trazo simple espiroideo. Panel B: radiografía antero-posterior y lateral de fémur izquierdo posquirúrgico inmediato de reducción cerrada y enclavado centro-medular con clavos TENS. Panel C: radiografía antero-posterior y lateral de fémur izquierdo, un año posterior a la reducción cerrada y enclavado centromedular con clavos TENS.
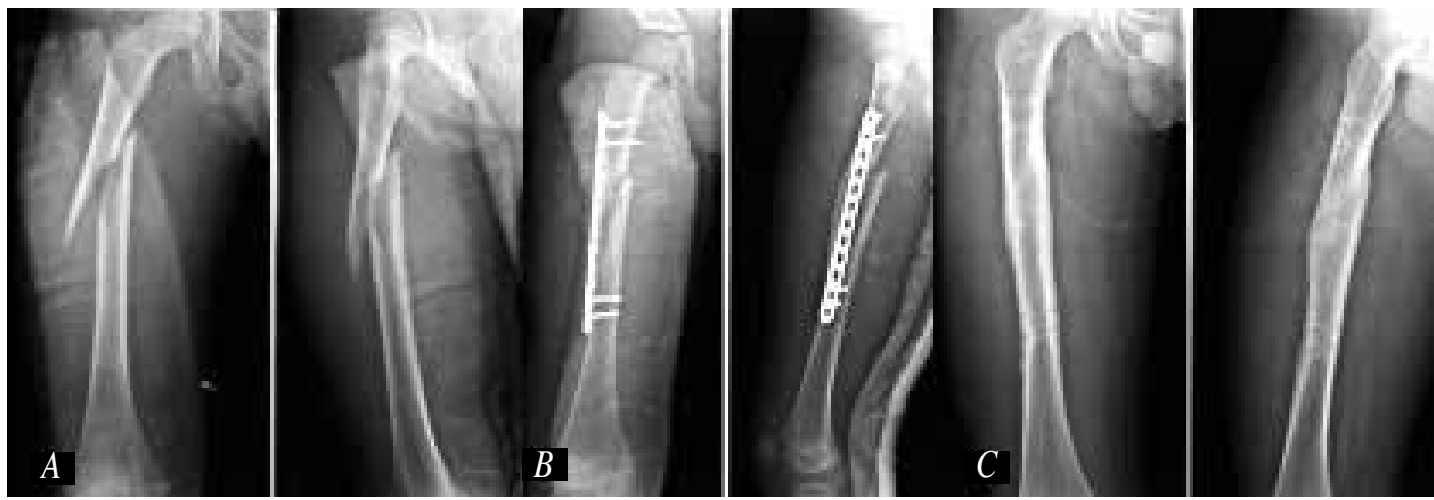

Figura 5. Panel A: radiografía antero-posterior y lateral de fémur derecho. Paciente masculino de ocho años de edad, con fractura diafisaria proximal. Panel B: radiografía antero-posterior y lateral de fémur derecho posquirúrgico inmediato de reducción abierta y fijación interna con placa LCP. Panel C: radiografía anteroposterior y lateral de fémur derecho en posquirúrgico inmediato de retiro de placa LCP, encontrando una consolidación grado IV de Montoya.

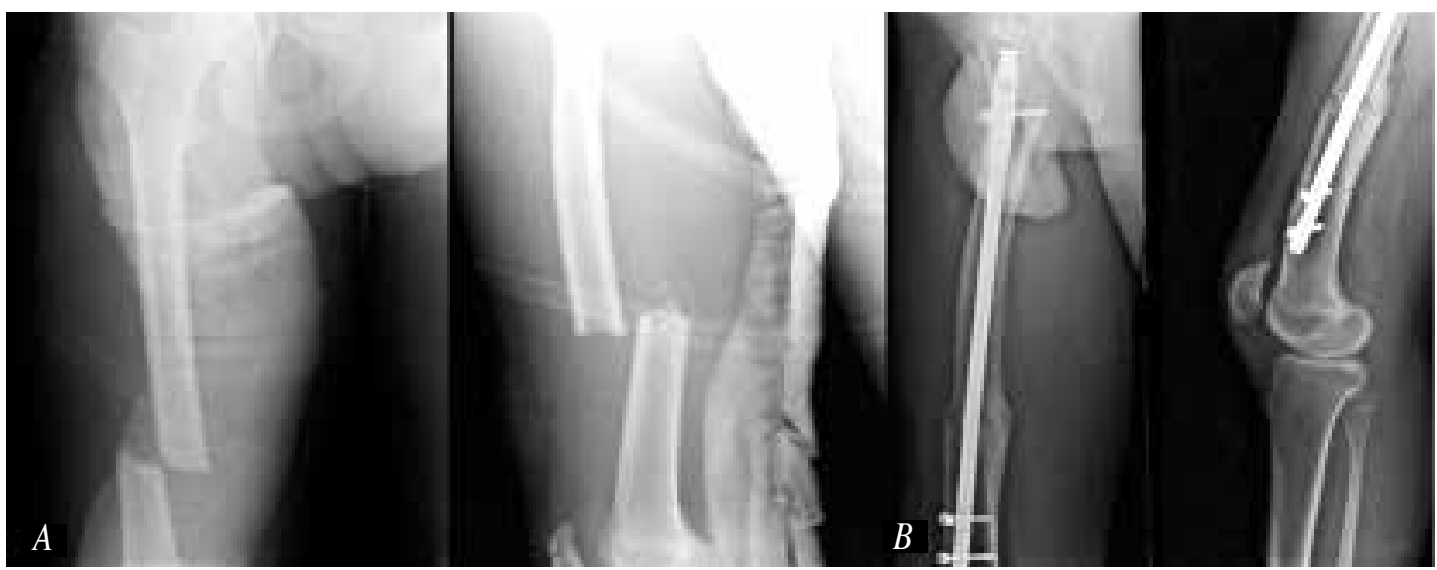

Figura 6. Panel A: radiografía antero-posterior y lateral de fémur derecho. Paciente masculino de 15 años de edad, con fractura diafisaria media trazo transverso. Panel B: radiografía antero-posterior y lateral de fémur derecho, cuatro semanas posteriores a la reducción cerrada y enclavado centromedular con clavo UFN. 
De los 52 pacientes ingresados al protocolo, solamente nueve de ellos el decir, el $17 \%$, recibieron tratamiento antitrombótico. En 36 de los pacientes (70\%) no se presentaron complicaciones, si se considera que una complicación temprana es la que se presenta en el primer mes posquirúrgico, que consiste en el cierre inadecuado de la herida, la infección del sitio quirúrgico $y$, una complicación tardía, posterior al mes posquirúrgico, como la osteomielitis y un acortamiento mayor de $2 \mathrm{~cm}$ de la ex- tremidad afectada. De las complicaciones reportadas en el 30\% restante, el 10\% correspondió a osteomielitis, otro $10 \%$ a pseudoartrosis y el otro $10 \%$ a infección de herida quirúrgica, exposición de material de osteosíntesis y fractura refractaria. La discrepancia de extremidades se presentó en ocho pacientes, que corresponde al $15 \%$ de la población estudiada, mientras que el $85 \%$ no la presentó. Cabe mencionar que el motivo de egreso de todos los pacientes fue por mejoría.

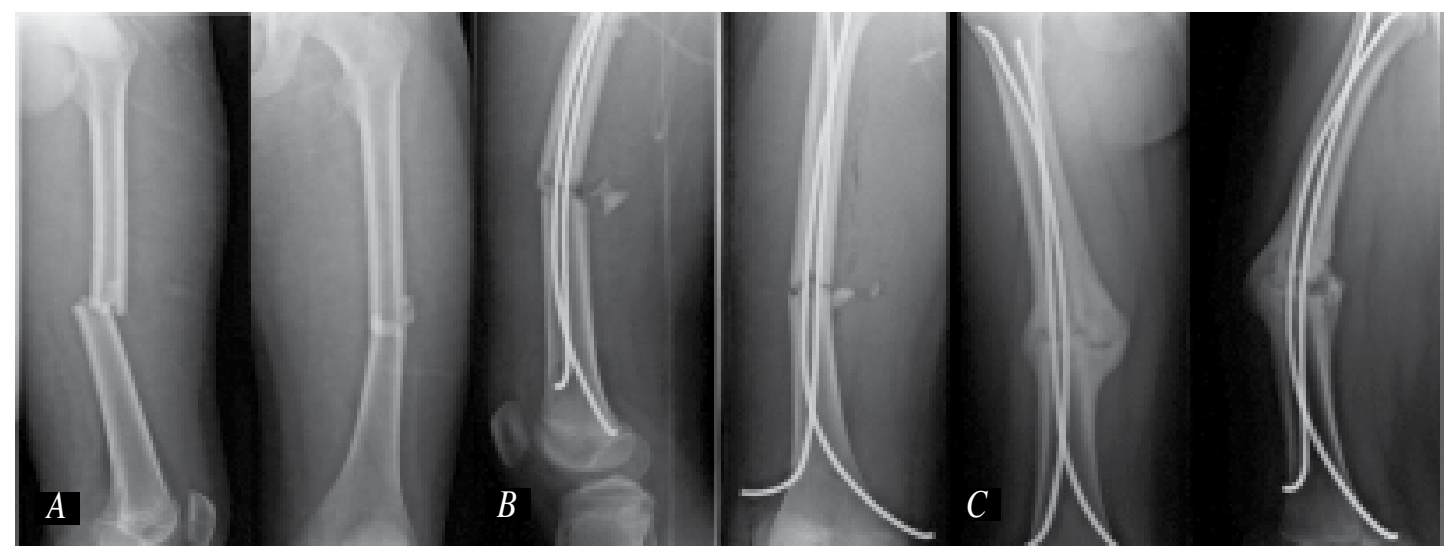

Figura 7. Panel A: radiografía antero-posterior y lateral de fémur izquierdo. Paciente masculino de 12 años de edad, con fractura diafisiaria media trazo simple transverso con tercer fragmento. Panel B: radiografía antero-posterior y lateral de fémur izquierdo posquirúrgico inmediato de reducción cerrada y enclavado centro-medular con clavos TENS. Panel C: radiografía antero-posterior y lateral de fémur izquierdo seis meses posterior a evento quirúrgico, se encuentra retardo en la consolidación.

| | | | | | | | | | | | | | | | | | | | | | | | | | | | | | | | | | | | | | | | | | | | | | | | | | | | | | | | | | | | | | | | | | | | | | | | | | | | | | | | | | | | | | | | | | | | | | | | | | | | | |

\section{Discusión}

Las fracturas diafisiarias de fémur en los niños sigue siendo un tema crucial para los especialistas en Ortopedia, debido a los variados tipos de tratamiento utilizados según la edad del paciente, el peso, tipo de trazo fracturario y las lesiones asociadas. ${ }^{4}$ En cuanto a la prevalencia que se encontró en el estudio fue de 3 por cada 1000 pacientes pediátricos que ingresaron por lesiones traumáticas y es ligeramente mayor a la reportada a nivel internacional, se estima que se debe a que el hospital es un centro regional de referencia. $1,5,7,13,16,20,22$
En el presente estudio se evidenció que los pacientes menores de seis años es el grupo de edad más frecuentemente afectado; a su vez, los pacientes menores de dos años fueron el pico máximo, lo cual coincide con el estudio realizado por Fuchs ${ }^{22}$ quien realizó un estudio con una casuística de 52 niños en la cual, en los pacientes menores de 6 años con este tipo de fracturas, el pico de edad de presentación era en los 3 primeros años de vida como el grupo más afectado. También encontramos un predominio por el sexo masculino siendo una relación de 3:1 lo que se acerca al estudio que rea- 
lizó Álvarez ${ }^{23}$ en el cual encontró que, de 59 pacientes estudiados, 47 pertenecían al sexo masculino. En cuanto al mecanismo de lesión, se evidenció que el trauma de baja energía fue el más frecuente siendo la caída de su propia altura un $23 \%$; esto concuerda con el estudio de Vega Fernández ${ }^{24}$ en donde un $48 \%$ se presentó con este mismo mecanismo de lesión, siendo menor el porcentaje en este estudio de traumatismo por alta energía entendiéndose por esto caída de motocicleta con un $15.4 \%$ o accidente automovilístico siendo sólo el 9.61\%. En cuanto al lado afectado, no hubo diferencia en lo reportado en la literatura ya que es similar la afectación tanto para el lado derecho como para el izquierdo.

Teniendo en cuenta que el tercio que se afecta con más frecuencia es el tercio medio siendo en este estudio el $65.4 \%$, seguido del tercio distal y por último el tercio proximal, lo cual asemeja al estudio realizado por Álvarez ${ }^{23}$ donde mostró que en un $64.4 \%$ de las fracturas se presentaban en el tercio medio, donde fue menor el porcentaje de exposición (11.5\%) al ser estos pacientes mayores de 13 años, asemejándose al cuarto grupo reportado por Hedström, el cual corresponde al 10.4\% en pacientes entre 13-18 años de edad. Los pacientes de este estudio recibieron en su mayoría un tratamiento conservador en un $54 \%$ a pesar de la variedad de trazos y mecanismos reportados, porcentaje similar al reportado en la literatura. En el 30\% de los casos, las complicaciones más frecuentes fueron la osteomielitis y la pseudoartrosis. El tratamiento quirúrgico se realizó en el $46 \%$ de los pacientes, se utilizó la reducción cerrada y el enclavado con clavos TENS (en el $44 \%$ de estos casos) fue usado en pacientes mayores de 5 años. En este grupo de pacientes, la principal complicación fue la seudoartrosis, en un 33\%. En cuanto a las secuelas, la discrepancia de extremidades se tuvo en un $15 \%$ siendo mayor que en el estudio realizado por Vega Fernandez ${ }^{24}$ quienes sólo reportaron un $7 \%$ en esta secuela al tener un universo de estudio similar al nuestro.

\section{Conclusiones}

Las fracturas diafisiarias de fémur en pacientes pediátricos sigue siendo un reto para el Ortopedista; sin embargo, el tratamiento conservador en pacientes menores de seis años ha mostrado excelentes resultados en el $\mathrm{CHMH}$ con el menor índice de complicaciones. En lo que respecta al tratamiento en el grupo de edad de 7 años a 13 años, se encontró una mayor dificultad para elegir entre un tratamiento quirúrgico o un tratamiento conservador, teniendo como pauta el trazo fracturario así como la lesión a tejidos blandos en caso de ser una lesión de alta energía; otra variable que no se tomó en cuenta por no contar con los datos en el expediente clínico, fue el peso del paciente, que es una variable que se tendría que tomar en cuenta al momento de seleccionar el implante, y en el grupo de edad de los pacientes mayores de 13 años la decisión fue menos complicada ya que en todos los pacientes realizamos un tratamiento quirúrgico. El implante a usar fue la disyuntiva del tratamiento ya que tuvimos que evaluar la fisis proximal para valorar si era posible la colocación de un clavo UFN. Aún se necesitan más estudios para poder evaluar correctamente las variables que nos darán la pauta para realizar el tratamiento óptimo dependiendo del grupo de edad, para así poder implementar medidas para disminuir el índice de complicaciones. En general, se consideró que los resultados obtenidos fueron satisfactorios en coincidencia con los trabajos que en los últimos años evaluaron los diferentes tratamientos para las fracturas diafisiarias de fémur en la edad infantil y adolescente. 


\section{Bibliografía}

1. M. Rapp M. M. Kaiser · F. Grauel - C. Gielok · P. Illing, Femoral shaft fractures in young children $(<5$ years of age): operative and non?operative treatments in clinical practice Eur J Trauma Emerg Surg (2016) 42:719-724.

2. Hedström EM, Svensson O, Bergström U, et al. Epidemiology of fractures in children and adolescents. Acta Orthopaedica. 2010;81:148-53.

3. Schalamon J, Dampf $S$, Singer $G$, Ainoedhofer $H$, Petnehazy T, Hoellwarth ME, Saxena AK. Evaluation of fractures in children and adolescents in a level I trauma center in Austria. J Trauma. 2011;71:E1925.

4. Madhuri V, Dutt V, Gahukamble AD, Tharyan $P$, Interventions for treating femoral shaft fractures in children and adolescents (Review) Cochrane Database of Systematic Reviews 2014, Issue 7. Art. No.: CD009076.

5. Flynn JM, Skaggs D. Chapter 22: Femoral shaft fractures. In: Kasser J, Beaty J editor(s). Rockwood \& WiIkins' Fractures in Children. Philadelphia: Lippincott Williams \& Wlkins, 2006:893-936

6. Gustilo RB, Anderson JT. Prevention of infection in the treatment of one thousand and twenty-five open fractures of long bones: retrospective and prospective analyses. Journal of Bone and Joint Surgery-American Volume 1976;58(4): 453-8

7. Gustilo RB, Mendoza RM, Williams DN. Problems in the management of type III (severe) open fractures: a new classification of type III open fractures. Journal of Traumalnjury, Infection and Critical Care 1984;24(8):742-6.

8. Slongo TF, Audigé L. Fracture and dislocation classification compendium for children: the $\mathrm{AO}$ pediatric comprehensive classification of long bone fractures (PCCF). Journal of Orthopaedic Trauma 2007;21(10 Suppl):S135-60.

9. Sameer M. Naranje, MD, ${ }^{*}$ Matthew G. Stewart, $M D$, wz Derek $M$. Kelly, MD et. al. Changes in the Treatment of Pediatric Femoral Fractures:15-Year Trends From United States Kids' Inpatient Database (KID) 1997 to 2012J Pediatr Orthop _ Volume 00, Number 00, 2015.

10. Heyworth BE, Suppan CA, Kramer DE, et al. Management of pediatric diaphyseal femur fractures. Curr Rev Musculoskelet Med. 2012;5:120-5.

11. Brousil J, Hunter JB. Femoral fractures in children. Curr Opin Pediatr. 2013;25:52

12. Moroz LA, Launay F, Kocher MS, et al. Titanium elastic nailingof fractures of the femur in children. Predictors of complications and poor outcome. J
Bone Joint Surg. 2006;88:1361-6.

13. American Academy of Orthopaedic Surgeons. Treatment of paediatric diaphyseal femur fractures. 2009. http://www.aaos.org/ research/guidelines/ pdffguideline.pdf.

14. AWMF-Leitlinie Femurschaftfraktur. German Guidelines of the AWMF Study Group. 2014. http://www. awmf.org/uploads/tx_szleitlinien/006-016I-S1_Femurschaftfraktur.pdf

15. J.-D. Métaizeau Fracturas diafisarias del fémur en el niño EMC - Aparato locomotor Volume $48>n ? 2>$ junio 2015

16. Dr. Rafael Serrano*, Dr. Gustavo Álvarez*, Dr. Enrique Vergara Amador** Papel del tratamiento ortopédico de las fracturas de fémur en los niños. Rev. Col. Or. Tra. 2009:23;(3):166 - 172

17. P. González-Herranz?, M.LI. Rodríguez Rodríguez y M.A. Castro Torre Fracturas diafisarias del fémur en el niño: actualización en el tratamiento Rev esp cir ortop traumatol. 2011;55(1):54-66.

18. González Herranz P, Rapariz González J, Burgos Flores J, Ocete Guzmán J, López Mondéjar J, Amaya Alarcón S. Femoral intramedullary nailing in children. Effects on the proximal end of the femur. J Bone Joint Surg. 1995;77-B:262-6, javascript: AL get(this, 'jour', 'J Am Acad Orthop Surg.'

19. Sanders JO, Browne RH, Mooney JF, Raney EM, Horn $\mathrm{BD}$,Anderson DJ, et al. Treatment of Femoral Fractures in Children by Pediatric Orthopedists: Results of a 1998 Survey. J Pediatr Orthop. 2001;21:436-41, javascript:AL get(this, 'jour', 'J Am Acad Orthop Surg.

20. Malkawi H, Shannak A, Hadidi S. Remodeling after femoral shaft fractures in children treated by the modified Blount method. Journal of Pediatric Orthopedics 1986;6(4):421-9.

21. Dameron $T$, Thompson $H$. Femoral shaft fractures in children. Treatment by closed reduction and double spica cast immobilization. Am J Orthop 1959; 41-A: 1201-12.

22. Fuchs $M$, Losch $A$, Noak $E$, Sturmer KM. Long-term results after conservative treatment of pediatric femoral shaft fractures. Orthopade. 2003;32(12): 1136-42.

23. Alvarez- Lopez A, Casanova Morote C, Garcia Lorenzo Y, Garcia Lugo F, Martinez Fernandez A. Fracturas de fémur en el niño comportamiento en cuatro años. Archivo Médico de Camagüey 2004;8(1) ISSN 1025-0255.

24. Vega Fernández E, Loredo QuesadaT, Tabío Fonseca $M$, y León Santana R. Tratamiento de las fracturas diafisiarias en el niño. Rev Cubana Ortop Traumatol. $2005 ; 19(2)$. 